\title{
Avaliação da Efetividade do Ensino em um Curso de Formação Continuada Semipresencial
}

\author{
Germano O. Ribeiro ${ }^{2}$, Thomaz E. V. Silva ${ }^{1}$, Albano O. Nunes ${ }^{1}$ \\ Francisca A. P. Pinto ${ }^{1}$ e F. Herbert L. Vasconcelos ${ }^{1,2}$ \\ ${ }^{1}$ Departamento de Engenharia de Teleinformática (DETI-UFC) \\ Grupo de Pesquisa em Telecomunicações Sem Fio (GTEL) \\ ${ }^{2}$ Universidade Federal do Ceará (UFC) - Instituto UFC Virtual \\ Campus do Pici, Bloco 901, CEP: 60.455-760 - Fortaleza - CE - Brasil \\ germanoribeirolo@gmail.com e herbertevirtual.ufc.br
}

\begin{abstract}
This paper presents the evaluation of distance continuing education courses in the Federal University of Ceará. To obtain information related to the course, an instrument for data collection was developed based on the methodology for assessing the effectiveness of teaching in the student's perspective. For data analysis, we seek to diagnose the quality of teaching in courses, identifying pedagogical factors that stood out positively or still need to be worked out by the best managers. The results indicate the feasibility of using the instrument developed, highlighting the students' opinion about 8 factors related to online tutors, presential tutors, course material and course participants themselves.
\end{abstract}

Resumo. Este trabalho apresenta resultados de avaliação dos cursos de formação continuada a distância da Universidade Federal do Ceará. Para a obtenção das informações relacionadas ao curso, um instrumento de coleta de dados foi desenvolvido baseado na metodologia de avaliação da efetividade do ensino na perspectiva do estudante. Para a análise de dados, buscamos diagnosticar a qualidade do ensino nos cursos, identificando fatores pedagógicos que se sobressaíram positivamente ou que ainda necessitam ser melhor trabalhados pelos gestores. Os resultados apontam para a viabilidade da utilização do instrumento desenvolvido, destacando a opinião dos cursistas sobre 8 fatores relacionados aos tutores online, tutores presenciais, material do curso e aos próprios cursistas.

\section{Introdução}

Atualmente, a informática é considerada um meio revolucionário nas relações humanas, principalmente a partir da popularização da rede mundial de computadores, a Internet. Com o constante aprimoramento da tecnologia, os computadores passaram a ter alta capacidade de processamento de dados, tornando-se equipamentos cada vez mais rápidos, menores e baratos. Diante disso, a incorporação do computador no âmbito educacional, como ferramenta didático-pedagógica, tornou-se inevitável e necessária. Portanto, a Educação não pode ficar a margem desta realidade, devendo apropriar-se dos avanços tecnológicos e incorporá-los à prática educativa [Valente 2002].

Através da mediação das Tecnologias da Informação e Comunicação (TIC's) e do acesso cada vez maior da população aos computadores e a Internet, a Educação à 
Distância no Brasil vem crescendo sistematicamente[Alves 2009]. Esse desenvolvimento provoca, entre outras reflexões, a possibilidade de melhoria da qualidade do ensino e o que pode ser considerado como adequado em termos de boas práticas pedagógicas e de gestão institucional para que o curso online tenha a mesma qualidade de um presencial.

Atualmente, o sistema Universidade Aberta do Brasil, programa da Diretoria de Educação a Distância (DED) da Coordenadoria de Aperfeiçoamento de Pessoal de Ensino Superior (CAPES), voltado ao ensino à distância conta com quase uma centena de instituições de ensino superior ofertando vagas de extensão, graduação e pós-graduação, distribuídas por todas as regiões brasileiras nesta modalidade de ensino [Alves 2009]. Percebe-se que tamanha dimensão fornece informações preciosas que sirvam de indicadores para viabilizar melhor qualidade de ensino na tomada de decisões administrativas dos gerenciadores desse processo.

Acompanhando a tendência no cenário nacional brasileiro de fortalecimento da EaD, o Ministério da Educação (MEC), tem disponibilizado recursos alocados diretamento na matriz orçamentária das Instituições Públicas de Ensino Superior (IPES) por meio da ação 30 20RJ destinados ao Apoio à Capacitação e Formação Inicial e Continuada de Professores, Profissionais, Funcionários e Gestores para a Educação Básica. Dentro deste contexto, a Secretaria de Educação Continuada, Alfabetização, Diversidade e Inclusão (SECADI/MEC) tem fomentado a oferta de diversos cursos nas áreas de Educação de Jovens e Adultos na Diversidade, Educação Ambiental Educação em Direitos Humanos, Gênero e Diversidade na Escola, além de questões ligadas a Educação do Campo, Educação Especial, Étnico-racial dentre outras, através de cursos em nível de extensão e especialização dirigindo-se principalmente a professores da educação básica.

A Universidade Federal do Ceará (UFC) ofertou três cursos de extensão em parceria com o projeto SECADI. Esse projeto tem como intuito qualificar professores em temáticas educativas, tais como: alfabetização de jovens e adultos; educação ambiental e educação em direitos humanos, tendo como público alvo professores das redes públicas estadual e municipal do Ceará. Para a avaliação dos cursos, utilizou-se a metodologia de avaliação baseada na avaliação da efetividade do ensino na ótica discente (do inglês Students' Evaluation the Theaching Effectiveness - SETE) [Marsh and Bailey 1993] adaptada a realidade do ensino a distância, tendo em vista, que essa modalidade de ensino oferece peculiaridades que não ocorrem em cursos presenciais, como é o caso das ferramentas síncronas e assíncronas e dos tutores presenciais e a distância.

Importante ressaltar que a avaliação é um processo de investigação que contribui para o aperfeiçoamento contínuo de pessoas e instituições envolvidas no processo de formação. Nesta pesquisa, foram extraídas, através de uma avaliação geral, informações relacionadas a opinião dos cursistas acerca dos seguintes fatores: tutoria, material didático, atividades, sistema de avaliação, tempo dedicado ao curso, dentre outros.

A dificuldade deste tipo de avaliação consiste em trabalhar uma grande quantidade de informações aliada a necessidade de se identificar os principais fatores que contribuem para a melhoria da qualidade e eficiência do curso. Isto posto, o presente trabalho propõe a validação dos dados obtidos com o instrumento de avaliação através da aplicação do alfa de Cronbach, e uma análise quantitativa das percepções dos cursistas de três cursos 
de extensão a fim de otimizar o processo de reestruturação administrativa e pedagógica dos cursos.

Este trabalho está organizado em seis seções: na Seção 2, apresentam-se os conceitos sobre avaliação e suas implicações na gestão de pessoas. Na Seção 3, serão apresentadas as políticas de formação continuada no Brasil e suas implicações na educação. Os aspectos metodológicos de caracterização e coleta de dados da pesquisa serão apresentados na Seção 4. Na Seção 5, faz-se a análise dos resultados e suas interpretações. Por fim, apresentamos na Seção 6 as considerações finais deste artigo e as perspectivas para sua continuidade.

\section{Avaliação Educacional}

A concepção de avaliação como simples indicador de desempenho, perde espaço nas instituições de ensino, à medida que a compreensão de aprendizagem se fundamenta na ideia de que ela é um processo crítico que visa o aperfeiçoamento da formação humana. Esta compreensão de avaliação permeia da literatura científica nos mais diversos pensamentos [Vianna 2000]:

Os resultados das avaliações não devem ser usados única e exclusivamente para traduzir um certo desempenho escolar. A sua utilização implica servir de forma positiva na definição de novas políticas públicas, de projetos de implantação e modificação de currículos, de programas de formação continuada dos docentes e, de maneira decisiva, na definição de elementos para a tomada de decisões que visem a provocar um impacto, ou seja, mudanças no pensar e no agir dos integrantes do sistema.

Assim como em [Silva et al. 2012]:

.... a avaliação da aprendizagem não pode ser concebida apenas como uma atividade racional e classificatória para se fazer comparações ou estabelecer um padrão, mas também identificar as transformações e aquisições de conhecimento de novas aprendizagens, por meio de um processo pelo qual se pode determinar em que medida ou intensidade tais mudanças de fato ocorrem.

Nas pesquisas recentes sobre a avaliação educacional busca-se a consolidação do ato de avaliar como uma ação reflexiva e integrada as questões pedagógicas, socioeconômicas e culturais de docentes, cursistas e gestores [Vianna 2000][Silva et al. 2012]. Além disso, a busca por uma metodologia que supere a simples disseminação de resultados que representa um instante do desempenho acadêmico ou institucional.

\subsection{Avaliação em EaD}

Embora seja cada vez maior o contato das pessoas com a EaD, assim como a experiência pedagógica acumulada por esta modalidade de ensino, estes avanços ainda são vistos com certa desconfiança tanto por parte do meio acadêmico como da sociedade [Alves 2011]. Isto se deve, principalmente, pelo fato de não entenderem as especificidades do modelo pedagógico e, consequentemente no processo de avaliação. 
Avaliações em Ambientes Virtuais de Aprendizagem (AVA) ocorrem sob os mais diversos aspectos, dentre eles, análises de fóruns [Bassani and Behar 2009], ensinoaprendizagem [Vasconcelos et al. 2008], aspectos metodológicos [Sales et al. 2008], dentre outras. Recentemente, no Brasil tem-se visto a aplicação de mineração de dados educacionais na EaD [Almeida and Pimentel 2010][Baker et al. 2011].

Portanto, o foco deste trabalho está relacionado com alguns aspectos importantes, como a participação dos cursistas nas atividades propostas, entrada no AVA, tempo de permanência em cada atividade, números de iterações nas atividade, dentre outros. Propostas de avaliação que envolvam mais de um aspecto simultaneamente ainda são recentes e pouco exploradas. Neste sentido, pesquisas apresentam uma técnica de extração de características denominada Análise de Componentes Principais, a qual é utilizada para avaliar a multidimensionalidade dos processos avaliativos em instituições de ensino superior [de Almeida et al. 2007][Silva et al. 2012].

Com o intuito de estender a percepção da dimensão humanista da educação [Gadotti 2007] destacando a concepção emancipadora da avaliação, esta pesquisa conta com conceitos da avaliação da efetividade do ensino através da metodologia SETE para extrair, a partir do olhar discente, as características mais relevantes da qualidade dos cursos avaliados [Marsh and Bailey 1993]. Essa metodologia foi completamente adaptada à realidade da $\mathrm{EaD}$, de modo que os fatores extraídos dessa aplicação estão sendo constantemente reavaliados de modo a consolidar uma prática de avaliação que sirva como guia para as futuras avaliações de larga escala dentro das universidades.

\section{Cursos de Formação Continuada no Brasil}

Observamos no Brasil, como política fundamental para a educação, a formação continuada de professores que, juntamente com a formação inicial, se destacam como possíveis formas de melhoria da qualidade das escolas públicas brasileiras.

Neste contexto, a escola desempenha vários papéis na sociedade atual, pois ela está inserida num contexto de constante mudança, e o professor assume um papel de motivador da mudança de atitude e pensamento dos cursistas [Portal and Franciscone 2007]. Ademais, o professor precisa estar preparado para os novos e crescentes desafios desta geração que nunca esteve tão em contato com novas tecnologias e fontes de acesso ao conhecimento, como hoje.

$\mathrm{Na}$ formação inicial do professor, possivelmente houve alguns aspectos deficitários, tais como [Oliveira and Nogueira 2002]: transmissão de conteúdos descontextualizados e/ou que evidenciam a memorização destes. Desta forma, a formação continuada surge como um amparo aos docentes de modo que eles possam vislumbrar e adquirir novas habilidades e competências para trabalhar os seus conteúdos nas salas de aula.

Neste sentido as IPES, com recursos provenientes do MEC por meio de suas secretarias, como por exemplo a SECADI, ofertam cursos no nível de aperfeiçoamento e especialização através da Universidade Aberta do Brasil (UAB), na modalidade presencial e semipresencial, por meio do Comitê Gestor da Política Nacional de Formação Inicial e Continuada de Profissionais da Educação Básica, instituído por meio da Portaria MEC $\mathrm{n}^{0} 1.087$, o qual visa apoiar a formação continuada de professores para atuar nas salas de recursos multifuncionais e em classes comuns do ensino regular em parceria com as IPES. 


\section{Metodologia}

Nesta seção, será apresentada os aspectos metodológicos da pesquisa, tais como: caracterização da amostra, instrumento de coleta de dados e a modelagem dos dados para a análise dos resultados.

\subsection{Caracterizando a amostra}

No ano de 2012, a UFC promoveu, em parceria com a SECADI, três cursos de extensão direcionados a capacitação de professores do ensino básico da rede pública estadual e municipal nas áreas de Educação Ambiental, Direitos Humanos e Educação de Jovens e Adultos, que totaliza 1242 professores envolvidos. No final desses cursos, tais professores foram convidados a participar dum processo de avaliação.

Participaram afetivamente da pesquisa 471 cursistas, ou seja, 37,9\% dos cursistas. Estes últimos de acordo com seus respectivos cursos, foram convidados a participar de uma avaliação do curso sobre aspectos relacionados, a atuação do Tutor online, do Tutor presencial, conteúdo do curso, atividades, dentre outros.

\subsection{Instrumento de coleta de dados}

Tabela 1. Descrição dos Fatores.

\begin{tabular}{|c|c|}
\hline Fatores avaliados & Descrição do Fator \\
\hline Aprendizagem Efetiva & $\begin{array}{l}\text { Está relacionado ao conhecimento adquirido ao longo } \\
\text { do curso por parte do cursista }\end{array}$ \\
\hline Entusiasmo & $\begin{array}{l}\text { Aponta se o Tutor online demostra dinamismo e inte- } \\
\text { resse no aprendizado do cursista }\end{array}$ \\
\hline Organização Tutor Online & $\begin{array}{l}\text { Mostra se o Tutor online teve o cuidado de preparar as } \\
\text { aulas virtuais e uma organização sistemática de suas } \\
\text { ideias }\end{array}$ \\
\hline Interação Tutor Online/Cursista & $\begin{array}{l}\text { Está associado a capacidade do Tutor online resgatar } \\
\text { a atenção da turma, trazendo-a para a discussão dos } \\
\text { conteúdos }\end{array}$ \\
\hline Relação Tutor Presencial/Cursista & $\begin{array}{l}\text { Apresenta se o Tutor Presencial estava sempre atento } \\
\text { e disposto a resolver os problemas dos cursistas }\end{array}$ \\
\hline Abrangência Conteúdo & $\begin{array}{l}\text { Está relacionado com o conteúdo do curso e como o } \\
\text { aprofundamento desse conteúdo é feito pelo Tutor on- } \\
\text { line }\end{array}$ \\
\hline Processos Avaliativos & $\begin{array}{l}\text { Esse fator está associado ao fato dos processos avali- } \\
\text { ativos terem sidos coerentes durante todo o curso }\end{array}$ \\
\hline Atividades/Trabalhos & $\begin{array}{l}\text { Relaciona as atividades/trabalhos do curso com a rea- } \\
\text { lidade do conteúdo apresentado, bem como sua apli- } \\
\text { cabilidade no contexto dos cursistas }\end{array}$ \\
\hline
\end{tabular}

Para que ofeedback dos cursistas pudesse ser sistematizado, foi desenvolvido um instrumento de coleta de dados, baseado na metodologia SETE. Tal instrumento apresenta 29 assertivas, avaliando 8 fatores que permeiam o processo de ensino na $\mathrm{EaD}$. Os cursistas avaliavam cada assertiva de acordo com uma escala Likert de 5 pontos (1- Discordo fortemente,..., 5- Concorfo fortemente). 
Os 8 fatores estão descritos na Tabela 1:

A aplicação do instrumento de coleta de dados se deu através de um endereço online e a respostas dos cursistas foram compiladas numa planilha eletrônica.

Os fatores apresentados acima, foram omitidos na aplicação do instrumento, pois a ideia era não deixar claro para o cursista os aspectos que eles estariam avaliando para não deixar os resultados tendenciosos por conta da explicitação dos fatores.

Por uma questão de espaço o questionário aplicado não será disponibilizado no corpo do artigo, entretanto poderá ser solicitado através de email para qualquer um dos autores.

\subsection{Modelando os dados}

Com um total de 13659 dados coletados, algumas estratégias foram adotadas de maneira que os dados pudessem ser analisados de forma clara e que não perdesse a robustez de informação contida neles. Para isso, os seguintes procedimentos foram adotados:

1. O alfa de Cronbach [Cronbach 1951] foi calculado item a item para atestar sobre a qualidade/confiabilidade dos dados;

2. Após a validação pelo alfa de Cronbach, uma média das assertivas de cada item foi realizada, reduzindo o número de variáveis para os 8 fatores;

3. E por último, foi extraída a média e o desvio padrão da informação dos 471 cursistas para que esses resultados pudessem ser analisados na Seção 5.

Os dados serão apresentados na forma de distribuição de frequência das respostas dos cursistas através de histogramas, e relacionados as médias dos fatores analisados.

\subsubsection{Confiabilidade dos dados}

Para calcular a confiabilidade dos fatores avaliados, foi utilizado o alfa de Cronbach [Cronbach 1951]. Esse indicador apresenta o quanto a respostas das assertivas de um determinado fator se assemelham, tendo em vista que os avaliadores utilizam a escala Likert. Um valor alto para o alfa de Cronbach $(>0.7)$ indica que as médias dos fatores representam de forma significativa a amostra [Cronbach 1951].

\section{Resultados}

Para que a análise dos dados pudesse ocorrer, o alfa de Cronbach foi calculado, e como apresenta a Tabela 2 podemos verificar que todos os dados obtidos com os 8 fatores analisados são confiáveis, pois demonstram ter seu alfa $>0.7$. Essa validação nos permite fazer inferência sobre as médias dos fatores, já que os dados se mostraram consistentes e confiáveis.

Além disso, pôde-se observar que todos os fatores foram bem avaliados, tendo em vista que estão com média $>4$. Isso indica que o curso foi de acordo com a expectativas dos cursistas, entretanto, destacamos a seguir alguns pontos que merecem observação.

$\mathrm{Na}$ Tabela 2, destacam-se os fatores Atividades/Trabalhos e Entusiasmo como aqueles que tiveram a melhor avaliação diante da percepção dos cursistas, ou seja, os tutores se mostram dinâmicos, procurando sempre a melhor forma de apresentar o conteúdo estudado. 
Tabela 2. Resultados obtidos com o instrumento de coleta de dados.

\begin{tabular}{c|c|c|c}
\hline Fatores avaliados & Média & Desvio Padrão & Alfa de Cronbach \\
\hline \hline Aprendizagem Efetiva & 4.5764 & 0.5578 & 0.8266 \\
\hline Entusiasmo & 4.5950 & 0.6502 & 0.8912 \\
\hline Organização Tutor Online & 4.5398 & 0.5902 & 0.7900 \\
\hline Interação Tutor Online/Cursista & 4.5791 & 0.6248 & 0.8997 \\
\hline Relação Tutor Presencial/Cursista & 4.4724 & 0.7860 & 0.9184 \\
\hline Abrangência Conteúdo & 4.4660 & 0.6577 & 0.9016 \\
\hline Processos Avaliativos & 4.3071 & 0.7060 & 0.7779 \\
\hline Atividades/Trabalhos & 4.6242 & 0.5487 & 0.7668 \\
\hline
\end{tabular}

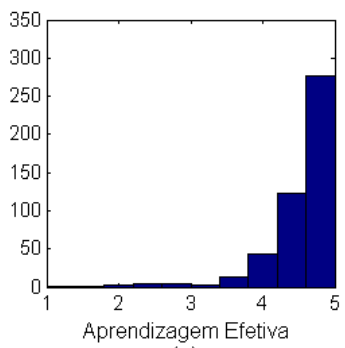

(a)

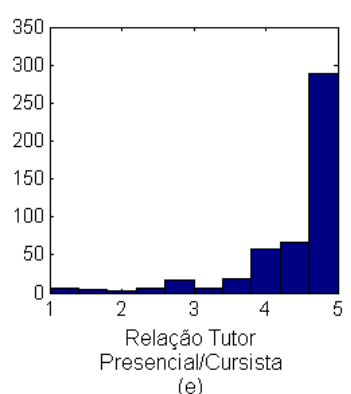

(e)

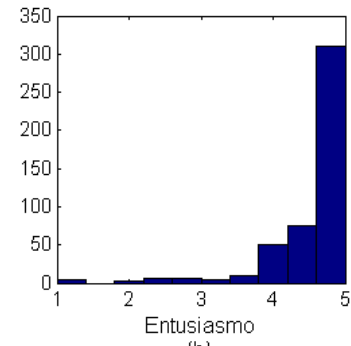

(b)

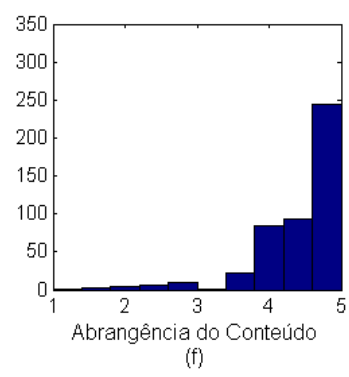

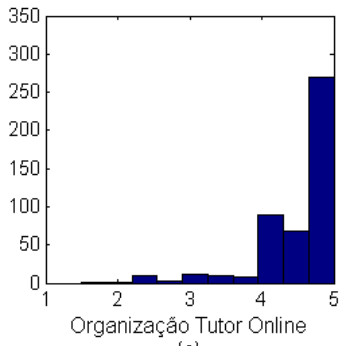

(c)

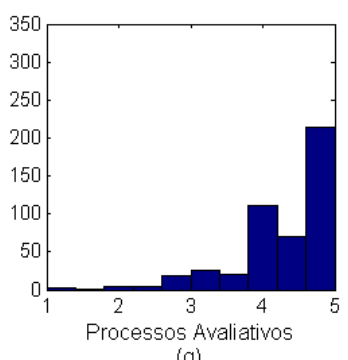

(g)

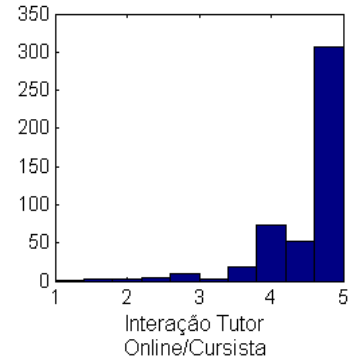

(d)

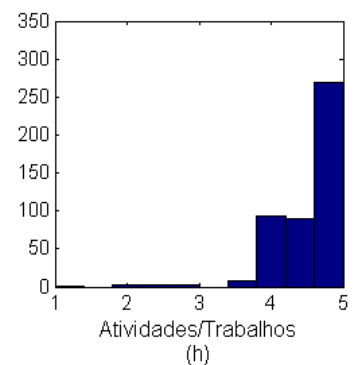

Figura 1. Distribuição de frequência das respostas dos cursistas

Importante ressaltar que os fatores Processos Avaliativos, Abrangência do Conteúdo e a Relação Tutor Presencial/Cursista ainda precisa ser melhorada. Ao investigarmos o por que das notas baixas para esses fatores nos deparamos com problemáticas como: o tutor online não deixou claro para os cursistas quais critérios de avaliação seriam levados em consideração durante o curso; o tutor online não aprofundou o conteúdo apresentado nas aulas virtuais, de modo que ele se restringiu apenas ao que já estava proposto como conteúdo programático dos cursos; e o tutor presencial não estava presente em alguns polos, o que dificultou a interlocução entre os cursistas e o polo que eles estavam alocados.

Uma outra forma de visualizar os resultados é através da distribuição de frequência das respostas dos cursistas, conforme é apresentado na Figura 1.

Na Figura 1 pôde-se observar uma dispersão, relativamente maior quando comparado aos outros histogramas, nas Figuras 1(f) e (g). Essa informação é importante, principalmente, para os gestores do curso, pois os cursistas sinalizam que os critérios/métodos de avaliação não estão claros e que os tutores online não aprofundam o conteúdo. 
Ainda na Figura 1, podemos identificar que as respostas, em todos os oito fatores gerados pela pesquisa, estão concentrados nos itens 4 e 5 da escala de Likert que versam respectivamente em concordo e concordo fortemente. Tal concentração remete a ideia de superestimar a avaliação do conteúdo pedagógico em virtude da utilização de ferramentas tecnológicas do curso. Esta hipótese toma parte das discussões, pois o contato inicial com novas tecnologias podem influenciar nas respostas dos participantes. A suposição corrobora com a percepção, através de conversas informais com os alunos, de que muitos deles tiveram contato pela primeira vez com um Ambiente Virtual de Aprendizagem durante a realização do curso.

Os dados analisados têm como objetivo melhorar os cursos avaliados dentro dos contextos apresentados anteriormente e, consequentemente o público alvo seja atendidos com maior eficiência.

Vale ressaltar que, durante o processo avaliativo de formação dos professores promovidos por esta instituição, alem do questionário avaliativo delineado nesta pesquisa, foram utilizados outras metodologias e mecanismos de acompanhamento do processo formação, por meio das seguintes ações: reuniões periódicas com os tutores presenciais e a distancia promovidas pelos coordenadores e supervisores dos cursos, curso de formação continuada para os tutores online, acompanhamento online do desempenho do tutor pelo seu respectivo supervisor.

Este trabalho apresenta algumas limitações, pois entendemos que a análise na forma de questionário não deve ser o único meio de avaliar a qualidade de cursos online, entretanto ela pode servir para sistematizar a informação proveniente da opinião dos cursistas sobre o curso avaliado. Portanto, este trabalho não pretende esgotar todas as possibilidades de se avaliar cursos online, e sim apresentar uma perspectiva simples e sistematizada sobre o objeto de estudo.

\section{Considerações Finais}

Neste trabalho, foi aplicado um instrumento de coleta de dados, baseado na metodologia de avaliação SETE, no contexto de cursos semipresenciais da UFC, esta aplicação teve como objetivo compor um banco de dados para análise dos cursos de extensão por meio da estatística clássica.

Comparando os resultados da Figura 1 com a Tabela 2, pôde-se observar a ocorrência de uma interseção da informação gerada pelas médias dos fatores com as distribuições das respostas dos cursistas para cada fator. Essa interseção foi validada pelo alfa de Cronbach.

A modelagem dos dados mostrou-se adequada para analisar o conjunto de dados gerados, tendo em vista os aspectos relevantes apontados pelos fatores estudadso, dentre eles destacam-se: deixar claro os critérios de avaliação das atividades; e exigir do tutor online um maior aprofundamento no conteúdo estudado para que ele não se limite ao que está nas aulas virtuais e etc.

O instrumento de avaliação fornece uma análise sobre todos os agentes que estão envolvidos no processo, como os cursistas, tutores online, tutores presenciais e gerenciadores dos cursos. Assim, torna-se importante ressaltar que as informações geradas por este trabalho servirão para melhorar/modificar os cursos de formação continuada oferta- 
dos pela UFC de acordo com as ações que já estão em andamento nos cursos.

A ideia de superestimar a avaliação do curso, apresentada na seção dos resultados, será objeto de investigação em trabalhos futuros. Tendo em vista a utilização de assertivas que contemplem a quantidade de cursos online que o aluno tenha participado até o momento da avaliação e se já fez uso da ferramenta avaliativa anteriormente. De tal modo que buscaremos compreender se há relação entre a quantidade de participações em cursos ofertados em Ambiente Virtual de Aprendizagem com uma análise mais criteriosa dos fatores que são investigados pelo instrumento avaliativo.

Como perspectivas futuras, pretendemos expandir a aplicação do instrumento para outros cursos, tanto no âmbito da pós-graduação quanto na graduação. A ideia é melhorar e aprimorar a avaliação no ensino a distância em adição ao que já é proposto na avaliação de tutores online, de modo que possamos verificar os erros para que eles sejam corrigidos.

\section{Agradecimentos}

Gostaríamos de expressar nossos sinceros agradecimentos à Secretaria de Educação Continuada, Alfabetização, Diversidade e Inclusão (SECADI/MEC) pela parceria com a Universidade Federal do Ceará, através do Instituto UFC Virtual.

\section{Referências}

Almeida, A. and Pimentel, E. P. (2010). Mineração de dados no ensino a distância para fins de avaliação do uso das ferramentas de aprendizagem do ambiente tidia-ae. In Anais do III WAValia.

Alves, J. R. (2009). Educação a distância: o estado da arte, chapter A história da EAD no Brasil, pages 9-13. Pearson Education do Brasil.

Alves, L. (2011). Educação a distância: conceitos e história no brasil e no mundo. Revista Brasileira de Aprendizagem Aberta e a Distância, 10.

Baker, R., Isotani, S., and de Carvalho, A. (2011). Mineração de dados educacionais: Oportunidades para o brasil. Revista Brasileira de Informática na Educação, 19.

Bassani, P. S. and Behar, P. A. (2009). Modelos Pedagógicos em Educação a Distância. Avaliação da Aprendizagem em Ambientais Virtuais. Artmed.

Cronbach, L. J. (1951). Coefficient alpha and the internal structure of tests. Psychometrika, 16:297-334.

de Almeida, T. L., Pinto, S. S., and Piccoli, H. C. (2007). Auto-avaliação na fundação universidade federal do rio grande: metodologia de avaliação. Revista da Avaliação da Educação Superior, 12(3):515-530.

Gadotti, M. (2007). Novas perspectivas para a educação. Number 41. Artmed.

Marsh, H. W. and Bailey, M. (1993). Multidimensional students' evaluations of teaching effectiveness: A profile analysis. The Journal of Higher Education, 64:1-18.

Oliveira, E. d. S. G. and Nogueira, M. L. d. L. (2002). Educação a distância e formação de professores; desafios e perspectivas. Revista Tecnologia Educacional, 31(157/158):95103. 
Portal, L. L. F. and Franciscone, F. (2007). Contribuições da educação continuada na construção da inteireza dos docentes da educação superior. Educação, 30(3).

Sales, G. L., Barroso, G. C., and Soares, J. M. (2008). O indicador de aprendizagem learning vectors como instrumento automatizado de avaliação para suporte a aprendizagem em ead. Anais do XXVIII Congresso da SBC.

Silva, T. E., Vasconcelos, F. H. L., Almeida, A. L., and Mota, J. C. (2012). Multivariate analysis for students' evaluation of teaching effectiveness in teleinformatics engineering. In Proceeding of IEEE TALE, Hong Kong.

Valente, J. A. (2002). O computador na sociedade do conhecimento. Campinas.

Vasconcelos, F. H. L., Cavalcante, A., de Oliveira, E. M., Sales, G. L., Almeida, R., Rodrigues, H. Z., de Castro Filho, J. A., and Pequeno, M. C. (2008). Avaliação sóciointeracionista aplicada ao contexto da ead em cursos de graduação semi-presenciais mediado por um ambiente virtual de aprendizagem. Anais do XXVIII Congresso da $S B C$.

Vianna, H. M. (2000). Avaliação Educacional: Teoria, Planejamento, Modelos. Ibrasa. 